\title{
International Manned Lunar Base: Beginning the 21 st Century in Space
}

\author{
Harlan J. Smith, a Alexander A. Gurshtein, ${ }^{b}$ \\ and Wendell Mendellc
}

The next generation of space vehicles will be capable of routinely carrying humans beyond the confines of the earth's gravitational field. Ambitious undertakings such as manned bases on the moon should greatly increase the scale and importance of constructive international interactions. At the present time the most appropriate arena for the closest cooperation in space seems to be scientific investigation. Achieving higher levels of cooperation in the grand schemes of future human exploration such as establishing human presence on Mars will require a buildup of interactions and trust through a series of programs of evolving complexity.

In this paper we present a part of the case for developing lunar bases, with detailed emphasis on the astronomical opportunities which they offer. Reasons are given as to why the development should be international, along with suggestions as to steps which can be taken in the next few years to undertake this initial level of largescale space cooperation.

\section{INTRODUCTION}

After three decades of manned space flight, there is no longer any serious question as to whether humans can or will ultimately move out into space. The real issues are who, when, where, why, what it will cost, and what should be the progression of this evolution.

\footnotetext{
a. Astronomy Department University of Texas at Austin and Lunar Planetary Institute. Houston

b. Academy of Sciences of the USSR, Moscow

c. NASA Johnson Space Center, Houston
} 


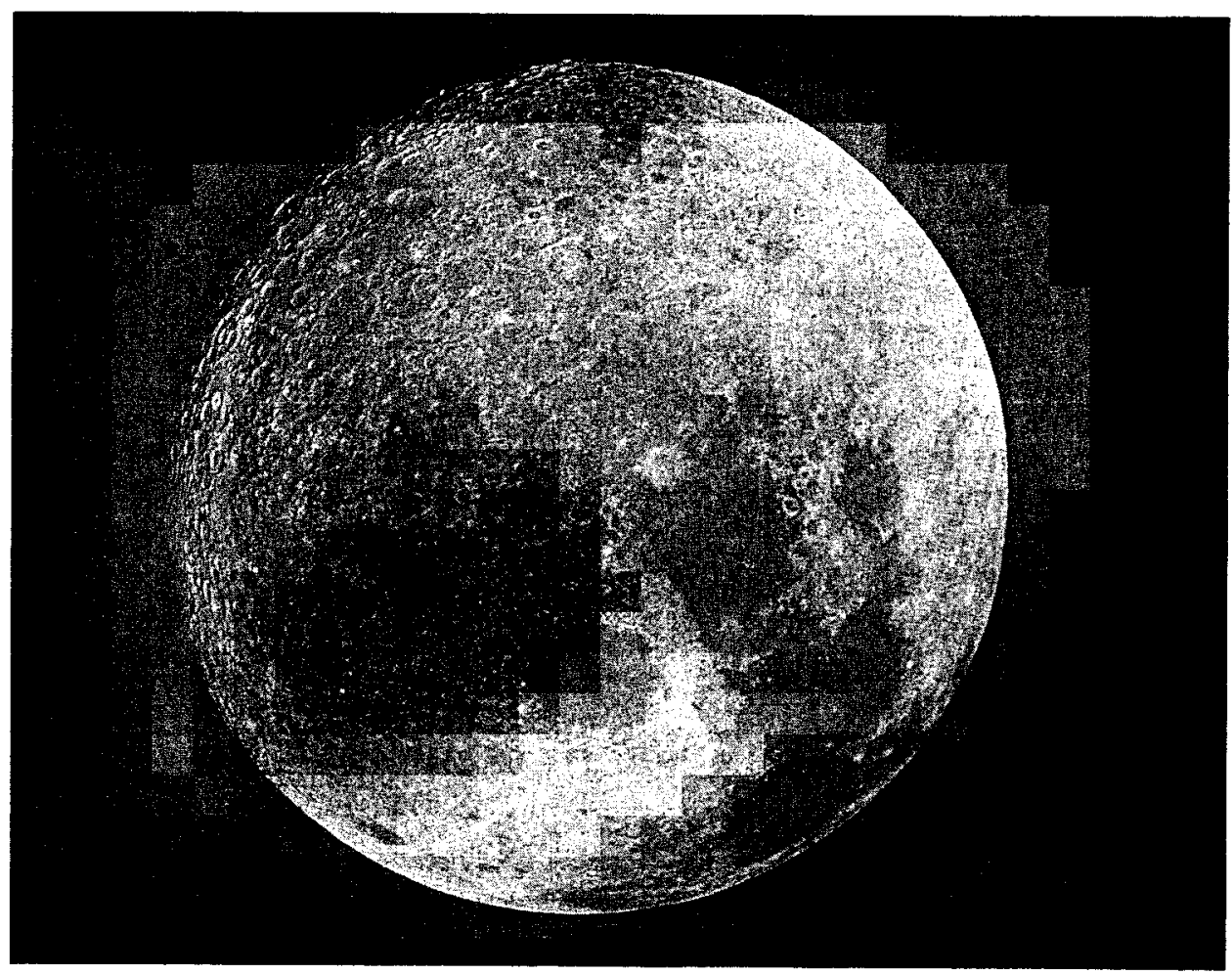

Figure 1: Spacecraft view of the western half of the near, earth-facing side of the moon, also including nearly half the side invisible from earth. The nearly circular dark feature below the center is Mare Crislum, and to its right is Mare Tranquillitatis, where the first human footprints on the lunar surface will probably be protected as a memory for all mankind.

Somewhat in the way that islands such as the Canaries were stepping stones to the subsequent opening of the Americas, this wonderfully situated satellite-a hundred times closer than any other major object in space-will serve many ends including that of base camp for human exploration and use of the solar system.

Source: NASA

Sufficiently reliable transportation systems now exist. The next logical stage in the human expansion should include several kinds of space station. The Soviets are leading the way with Mir, and there is reason to hope that before the end of the century there will be at least one additional station built by the US, the European Space Agency (ESA), and Japan.

Planning has of course already begun for manned-space-flight goals to follow the space stations. For the first half of the next century there remain only two serious possibilities-the moon or Mars.

For more than a century visionaries have discussed flights to the moon. 
The vision became reality with Korolev's adaptation of Soviet ballistic missiles for the first unmanned flight to the moon and the essentially simultaneous development of similar programs in the US. These investigations were welcomed with enthusiasm by the international scientific community and culminated in the six Apollo manned landings between 1969 and 1972. Nevertheless, even as the Apollo program continued, opponents began to challenge both the advisability of manned flight to the moon and even the moon itself as the target of further scientific study. Thus, for example, the astrophysicist Fred Hoyle in his book Galaxies, Nuclei, and Quasars expressed doubt as to the advisability of expending large sums on studying the "heaps of rock which the Moon is."

By the early 1980s interest in the moon had indeed diminished both in the US and in the Soviet Union. Recent efforts and achievements in space have related to Venus, Mars, Halley's Comet, the giant outer planets, and their satellites. In particular, the study of Halley's Comet stimulated fruitful interactions within the framework of large-scale international space collaboration.

More recently, however, the big leap in long-duration earth-orbital Soviet manned flights and the revival of the US space shuttle program are renewing a debate that was particularly prominent two decades ago, before the United States adopted the space shuttle program. At that time a manned lunar base and/or a piloted mission to Mars were considered as reasonable successors to Apollo.

The case for Mars is very strong. It offers the most exciting challenge. Mars is a planet with many fascinating mysteries. It will be the easiest albeit still difficult place for humans to set up large-scale housekeeping, having all the raw materials needed for ultimate human development. Thanks to an effective campaign, mounted principally in the US by the Planetary Society but with supporters in other countries as well, the idea of an early international manned expedition directly to Mars has gathered considerable momentum and some political backing. Attention was drawn to this proposal in the summer of 1988 by General Secretary Gorbachev during the Moscow summit meeting with President Reagan.

However, the problems in tackling Mars with human expeditions even a decade from now are serious. They include its great distance, the still relatively primitive state of our space technology, the extreme hazard of the 
undertaking, the limited scientific increment that would come from early missions (in the face of the rich global and site-specific data already gathered by then through orbiters, robotic landers, and sample return missions), and the exceedingly high cost along with the need for yet much larger classes of expenditure over a very long period of time before there could be any slightest prospect of economic return. It should be recalled that Mars at even its nearest approach is more than 100 times farther than the moon. This translates rather directly into travel time. The moon can be reached in several days, whereas round trips to Mars will take from one to several years. While there is less difference in energy requirement, since most space travel is simply coasting in orbit, the costs of going to Mars are still several times greater than for going to the moon, and the life-support and reliability requirements are at least an order of magnitude greater. Even communications are sharply different. Round-trip conversations with the moon have only a three-second lag; with Mars the interval between question and response ranges from about five minutes to half an hour. Medical or other emergencies that can be handled on the moon would be fatal on Mars missions where astronauts will be strictly on their own for up to three years. Many of these points have been presented in the Ride report (1987).

While we support manned activity on Mars as a longer-range goal, we believe that to make it the immediate next target is not only premature but is likely to create a situation in which the high costs, the all-too-possible failures, the absence of economic payoff, and even, given a success, the "what do we do next?" syndrome could lead to a backlash similar to that which followed the US Apollo program. The growth of such an attitude could severely weaken longer-term world space activity, especially if an adequate infrastructure to support a broad range of space functions has not been built up along the way. While this may be more likely to occur in the US, it could also happen in the Soviet Union as the growing power of public opinion and the desire to see more money spent on terrestrial problems and domestic development to play ever larger roles over the next several decades. President Kennedy's decision to go directly to the moon was a bold political move but was by no means the optimum engineering and economic path for human expansion into space. A rice has been paid for this choice, and we would be reluctant to see it repeatd with Mars.

For these, and other reasons to be discussed, we believe that the manned 
lunar base should be the focus of a new international collaboration in space. Such would make sense even if a mission to Mars remained one's principal goal. A great deal of additional experience is needed to learn how to build and operate efficient and effective, yet affordable, long-term life-support systems under conditions of relatively low gravity and virtual absence of atmosphere. The moon is the obvious place for this experience to be gained before committing people on the first expedition to Mars, where they would have to utilize these skills to survive on the surface for a considerable time before undertaking the return flight. We contend that only on the lunar surface can such experience be obtained on a full and relevant scale.

In addition, there are many reasons why lunar development is important for its own sake.

\section{THE FUNCTIONS OF A MANNED LUNAR BASE}

\section{Lunar Exploration}

Investigations on the surface of the moon, which is eroded neither by atmosphere, hydrosphere, nor biosphere, are important to our understanding of the origin and evolution of the solar system. Geologists, who rarely encounter ancient terrestrial rocks, can track only with difficulty evolutionary processes on the earth that took place prior to the past one or two billion years, but even the first lunar samples yielded information on events that had taken place in the solar system three to four billion years ago. A number of lunar continental provinces with quite complicated geology may well be contemporaneous with the "Day of Genesis" and could, according to modern theories, reflect the period of the formation of the earth's present crust.

The last three decades of lunar investigation have yielded important astronomical, geochemical, and geophysical measurements, generating significant scientific results: for example, proof that the earth and the moon are essentially the same age; discovery of asymmetry between the near and the far sides of the moon; absence of a global lunar dipole magnetic field; identification of basalts in the maria and anorthosites in the highlands; detection of large subsurface mass concentrations (mascons); and what appear to be traces of ancient magnetism in lunar rocks.

It is now known that the early evolution of the moon was similar to that of the earth, and that key fact underlies the modern cosmogonic model of the 
origin and the evolution of the whole solar system. For thousands of years of human history the only object for geosciences was the earth. This absence of planetary perspective prevented scientists from creating a general theory of the earth that could be tested. But today the situation has drastically changed. Modern lunar data coupled with results from exploration of other planets gave rise to the discipline of comparative planetology, which should help us discover and develop natural resources on earth while maintaining the natural environment.

Scientific facilities on the lunar surface, operated partly remotely, partly by scientists on site, offer the earliest opportunity to generalize our ideas about planetary phenomena through long-term observation coupled with the uniquely human ability to react to unexpected results and phenomena. These scientific facilities would study the earth, the sun, other bodies of the solar system, the interplanetary medium; address questions of cosmology; and make astrophysical observations as well as probe the lunar surface.

Initial lunar exploration should contribute to a better understanding of the relative roles of endogenous and exogenous factors in successive stages of the formation and evolution of a planetary body. Such selenological problems will be addressed utilizing the same research techniques used on the earth, specifically field geology, geophysics, and geochemistry. Data obtained in the field could then be extrapolated to much of the lunar surface with the help of orbital surveys using electromagnetic and gamma-ray remote sensing, geophysical measurements, and mapping of other regional properties.

The retrieval of lunar subsurface samples by means of deep drilling would be a major goal of lunar scientific exploration. An eventual target for the operation should be depths of one to two kilometers at several sites. This will require the development of novel techniques for dry vacuum drilling and removal of cuttings, some of which may find terrestrial applications.

Many of these same technologies will be vital in developing lunar resources for eventual commercial application. Among the strategies which may have large-scale potential are the extraction of oxygen from lunar materials for use as a rocket propellant, the production of metals for space structures, and the mining of helium-3 to serve as a fuel for low-pollution fusion power reactors on earth. The moon's low gravity and lack of atmosphere make it potentially far easier to export matter from there to space, as opposed to shipping it up from the earth. 


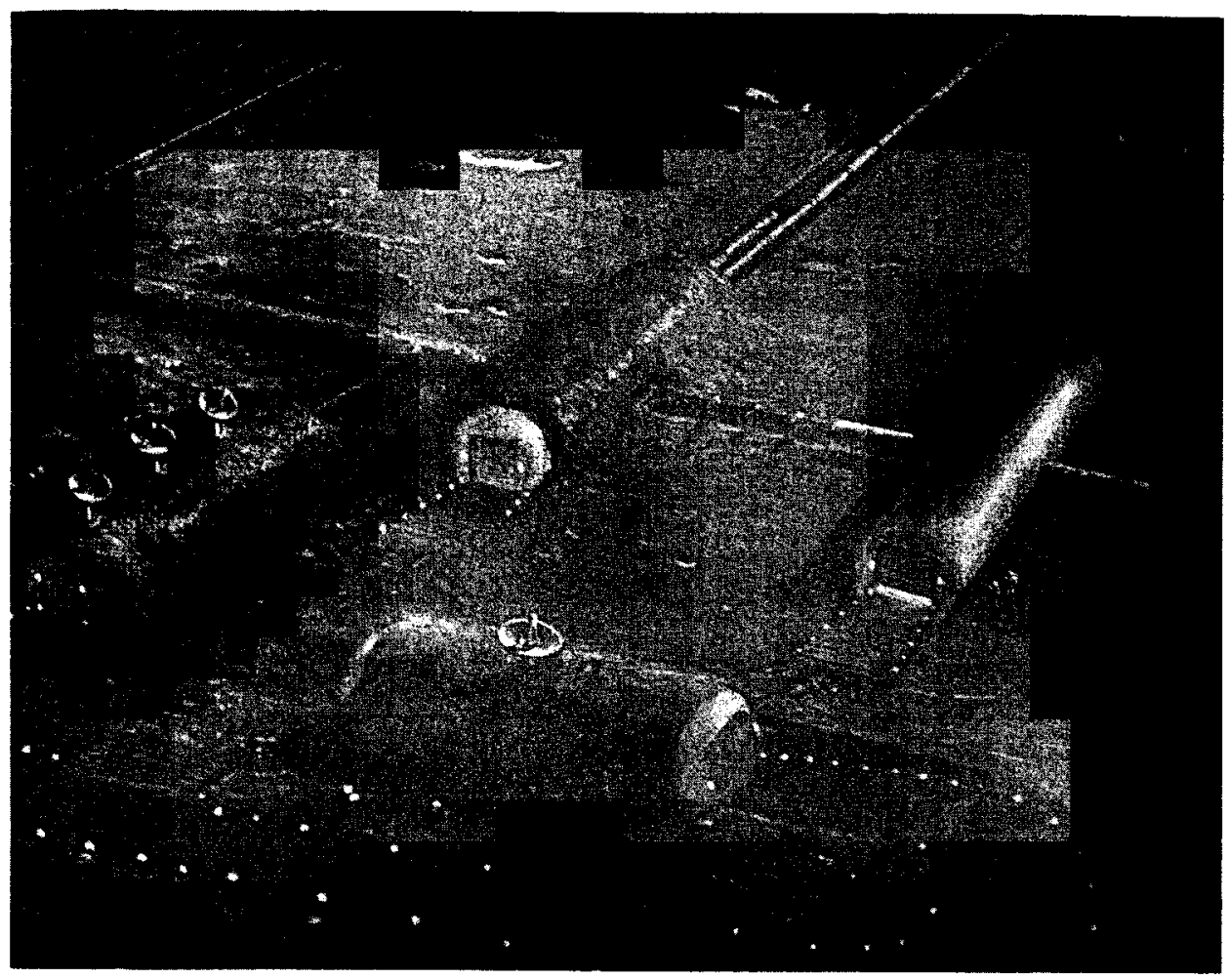

Figure 2: Possible appearance of a materials exporting site. operating with solar power during the two-week-long lunar day. Products such as oxygen fuel may be ejected into lunar orbit with a mass-driver (electromagnetic sled). All living and working areas must be buried under several meters of materials such as the lunar regollth, in order to protect inhabitants against the cumulative effects of cosmic rays.

Source: NASA

\section{The Lunar Environment with Respect to Astronomy}

An extensive literature (for example, Mendell 1985, Johnson and Wetzel 1988) deals with the above and similar topics. However, a major application of moon bases-serving as sites for astronomical observatories-is less widely recognized as yet. Because of our own scientific interests, and because this promises to be so important in the scientific development of the 21st century, we choose to present the case in some detail as a paradigm for what in time will develop in a number of fields of science and technology. The advantages of lunar observatories have also been forwarded in a recent article by Burns et al. in Scientific American (1990). 
The most important characteristics of the lunar environment for astronomy include:

- Ultra-high vacuum. The total mass of the lunar atmosphere is about the same as that of the air in a terrestrial movie theater (Taylor, 1988). Diffused over the entire lunar surface, this leads to a density comparable to the finest vacuums attainable in terrestrial laboratories. So low a density means that for all practical purposes the lunar surface is in free space, allowing unaffected observations to be made in all wavelengths other than perhaps the very lowest frequency use in radio astronomy. Telescopes can be used with their full spatial resolving power. Radio, infrared, and optical interferometers can be established with baselines of up to tens or even hundreds of kilometers. The lack of atmosphere means that exposed optical surfaces should retain their full efficiency for very long times, limited only by micrometeorite effects.

- Stable solid surface. It is possible to orient free-flying instruments with very great accuracy, but it is also rather hard to do so. This is one of the principal factors making space telescopes so very expensive to build and operate as well as substantially cutting into their effective observing time, and the problem will only become greater as larger instruments are needed. Conversely, the moon offers a solid platform with effectively infinite inertia, allowing simple low-cost terrestrial-type mountings to be used. Its rigidity and minimal seismic activity ( $10^{-8}$ that of the earth) ensure stability, again at very low cost, for elements of interferometers with even the longest spacings.

- Dark sky. The moon should be virtually free of the air glow (a kind of permanent aurora) that sets a limit on the darkness of even the darkest terrestrial nights. The absence of atmospheric scattering means that the deepest observations can be made at night even when there is a "full earth" -a hundred times brighter than the full moon in our sky. Indeed, with proper shading of telescopes it is likely that relatively deep observations can be made in the daytime. Under the best conditions, terrestrial and near-earth-orbit telescopes are collecting data only about a quarter of the time, whereas their lunar counterparts should seldom stop observing. 
- Cold sky. One of the most important spectral regions, only now beginning to be seriously opened up by astronomers, is the so-called thermal infrared. These long infrared wavelengths are generated by every object not at absolute zero, and the amount of this radiation increases very rapidly with temperature. Observing at such wavelengths is like trying to do optical astronomy using a telescope every element of which is emitting brilliant light, while looking through a brilliantly self-illuminated daytime sky. So far, the best solution is to use liquid helium to cool every part of the telescope as close as possible to absolute zero, and to put the system above as much atmosphere as possible using balloons or spacecraft. All this is extremely expensive to do even with small telescopes and also either suffers from limited lifetime of the helium cryogen supply or demands expensive and difficult resupply. On the other hand, the temperature of the sky as seen from space or the moon's surface is only a few degrees above absolute zero. A telescope on the moon-if carefully insulated from the ground and shielded from any direct or reflected radiation not coming from the dark sky-would cool to and remain at an exceedingly low temperature in the area of $73 \mathrm{~K}$. This would greatly reduce and perhaps in some cases eliminate the need for cryogens, as well as allow the telescope to be quite large.

- Low gravity. Experiencing only one sixth of the earth's gravity, lunar structures of any size will be of much lighter, less expensive construction than their terrestrial counterparts. This modest lunar gravity, however, serves the useful function of causing debris and contaminants to fall to the surface, rather than tagging along in place as they tend to do in space.

- Absence of wind. We often forget how much of the strength built into terrestrial systems is simply insurance against the threat of extreme wind forces, no matter how unlikely. On the moon, structures can be built with attention purely to static and thermal loads. For example, telescope “domes" might normally be simple systems of movable sun-, earth-, and dust-shades probably consisting of multiple layers of virtually weightless aluminized foil, stretched over ultra-lightweight but rigid structures. Telescopes themselves, which on earth must be carefully strengthened against low-frequency vibration resulting from wind-buffeting, will be able to concentrate solely on static forces at various angles. 
- Rotation. The moon's roughly month-long rotation period guarantees access to all the sky visible from the latitude of the observatory site, yet is slow enough to permit very long integrations on the faintest possible objects. Some of the most important modern observations demand unbroken time series with durations of weeks or longer, which are extremely hard to get from the ground or anywhere else except deep space. The rotation also gives free sky-scanning to very large telescopes and the possibility of aperture synthesis to interferometers.

- Proximity to earth. Since the moon is close enough to earth for round-trip communication times to be less than three seconds, some degree of earthbased control of robotic systems will be reasonable. This time lag offers no hindrance at all to giving astronomers on earth detailed real-time control of observing programs. Likewise it is trivial for massive data streams to be continuously broadcast to the earth for analysis. In other words, few if any astronomers will need to be on the moon, even when a great many telescope systems are functioning.

- Distance from earth. Human activities, plus those of the earth itself, generate noise and interference in nearly all possible kinds of observations. At 400,000 kilometers, the moon is far enough away to be relatively well quarantined from most of this pollution-experiencing a hundred-fold reduction below the levels even at geosynchronous orbit (40,000 kilometers). Except in certain radio frequency bands, observations from the moon will be virtually unaffected by the presence of the earth in the sky.

- The lunar farside. Some types of optical observations are approaching the stage of being able to detect and utilize a large percentage of all the information entering the telescope at the wavelengths of interest. However, this has not been possible in most of the bands of interest to radio astronomers because of terrestrial broadcasting and other interference. This is unfortunate, since radio astronomy is fundamental to astrophysics research and also comprises the part of the electromagnetic spectrum in which there are reasons to believe that extraterrestrial civilizations would be most likely to produce detectable emissions. For limiting sensitivity, radio astronomers must have a site which never sees the earth in its sky. Only one place in the universe realistically satisfies this condi- 
tion-the back side of the moon. It is vital for this unique state of radio quiet to be preserved as lunar development proceeds.

- Raw material. The moon offers an almost inexhaustible supply of many essential materials. In the beginning the raw lunar regolith will form essential shielding for selenauts against solar and galactic cosmic rays as well as outstandingly good insulating material. As processing facilities gradually come into operation, various cements and building blocks, then ceramics, glasses, fibers, and metals will become available. These will be increasingly important as very large astronomical instruments are eventually undertaken.

- Landforms. Perhaps the most cost-effective radio telescope ever built is also the world's largest-the 300-meter dish lining a hemispherical crater near Arecibo, Puerto Rico. On the lunar surface, beautifully symmetric craters come in almost any desired size. It is reasonable to expect that, aided by the low lunar gravity and lack of wind and weathering action, similar radio telescopes up to kilometers in diameter are likely someday to be built on the moon.

- Room. The moon offers effectively unlimited area for laying out systems of instruments, which can be added to at any time, and yet be conveniently located at one or more common bases for supplies of consumables, replacement parts, electric and computer power, etc.

- Access. The last, but perhaps most important, lunar advantage with respect to other kinds of astronomy from space will be the immediate proximity of people and support facilities. For the first time this will allow construction of major, highly sophisticated space telescopes and instrumentation in extremely simple, low-cost mountings and housings, with virtually all components readily accessible for maintenance or change by skilled people in the immediate vicinity. Nearly all the observing will be done by astronomers on earth, but the continuous availability of real-time, handson technical support will constitute a revolution in the way cost-effective space astronomy can be done.

Many hundreds of millions of dollars each year, worldwide, go into the constant effort to build and use better telescopes at better sites. This activity began seriously in the last century with the construction of ever-larger tele- 
scopes and their removal first to sites outside cities, then to high hills, next to towering peaks in remote deserts or islands, and recently into near-earth space. Quite apart from the many other factors impelling the human race in that direction, the steady unfolding of this tradition should soon lead us to the moon-the best place in the solar system from which to do many kinds of astronomy.

\section{SITING AND DEVELOPMENT OF THE LUNAR BASE WITH PARTICULAR REFERENCE TO ASTRONOMY}

\section{Siting of a Lunar Base}

Ultimately there must be many lunar bases. However, in the time frame of the next two decades, of greatest interest to this article, we may expect only one or at most two. Accordingly it is necessary to take into consideration

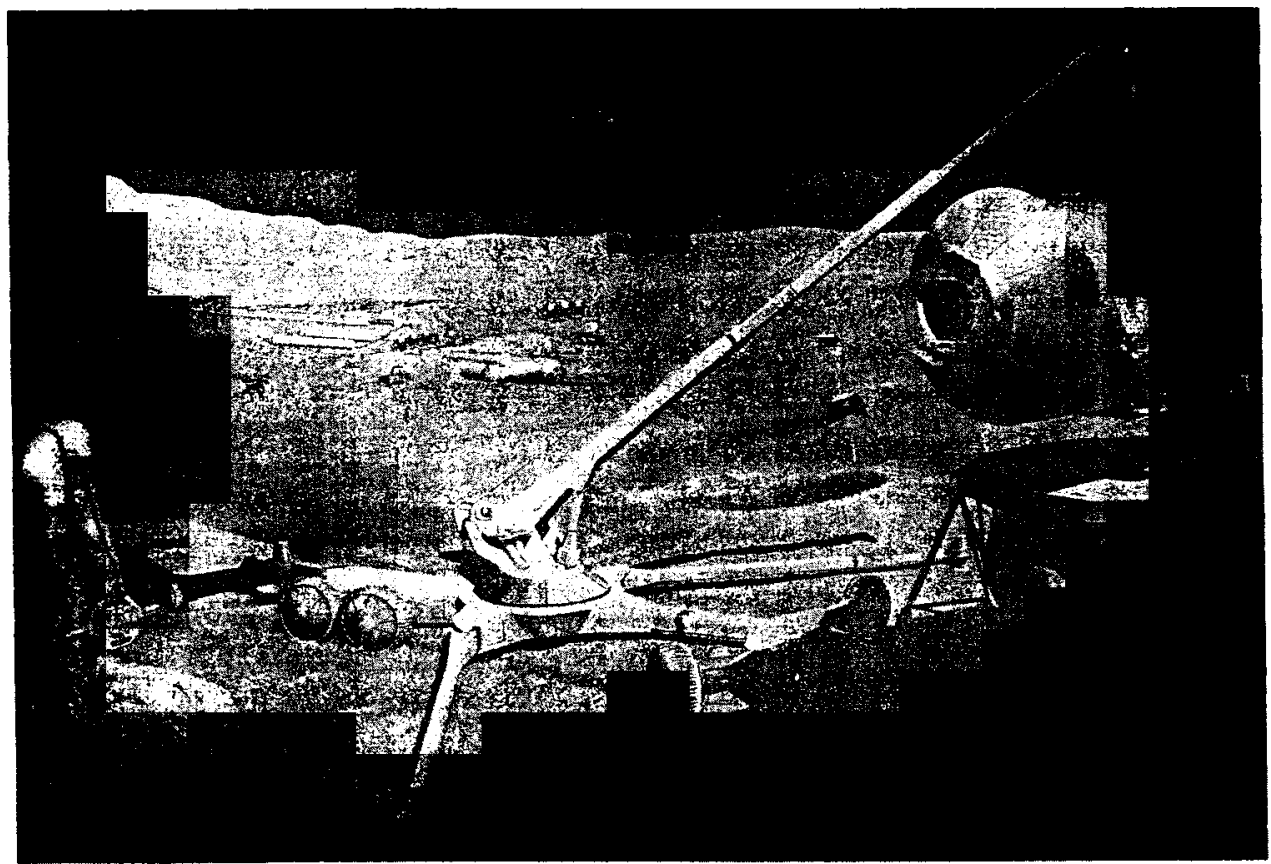

Figure 3: Seen from any spot on the near side of the moon. the earth is always about the same place in the sky. In the case of this base located near the western lunar limb. it is always found low on the eastern horizon.

Establishing a significant lunar base will require a number of unmanned cargo-ship landings. Low lunat gravity, only one sixth that of earth, helps substantially, but simple machinery will still be needed to handle large masses. 
some conflicting factors in the siting of the initial base(s). The desiderata that the earth be observable from the lunar base and easily communicated with effectively require that the base be on the earth-facing (visible) side of the moon. On the other hand, the need for totally dark night sky (simplifying shielding against scattered light) for radio astronomy of the very faintest astronomical objects requires access to the back side where the earth is never visible in the sky. Thus, the astronomically best location may be in the marginal zone of the lunar visible portion, where the earth can always be seen from the station but where relatively nearby optical and infrared telescopes can operate in the earth-shadow of hills or the rapidly curving lunar horizon. However, because of diffraction effects at the horizon and small libration effects changing slightly the position of the earth in the sky, a very low frequency radio astronomy array would need to be many degrees into the back side in order to be effectively shielded from the earth.

Given only a single site, astronomers would wish to be near the lunar equator so as to gain observational access to almost the entire celestial sphere.

Lunar exploration would benefit from a location near a geologically complicated province typical of the lunar surface. But spacecraft operations and construction simplifications suggest that the site should lie on a plain. The best choice may well be the margins of a mare, where the mare basalts contact the highland materials, thereby giving access to a variety of mineral resources. While other considerations will of course also enter into the decision, it is interesting that the vicinity of Mare Orientale satisfies all the above criteria and will surely be a strong candidate for the initial site.

\section{Phasing of Development}

Development of lunar bases in the 21st century will fall into three broadly distinguishable phases, similar to those required in the settling of any radically new region.

\section{Pioneering Phase}

First comes the pioneering phase, when nearly all the concentration is simply on getting there and surviving. During this time relatively little load capacity and attention are likely to be spared for science. Nevertheless, even the initial return to the moon will mean a continuation of geological and cosmogoni- 
cal investigations as well as the beginning of active programs on human physiology, probably also on experimental raising of plants and animals towards eventual partial self-sufficiency of food supplies.

Astronomy should play a major role from the beginning. A plausible scenario, though of course far from unique, for at least the earlier phases of this development, might be as follows: the first return landing should carry two very modest telescopes. One would be a fixed transit telescope, having an

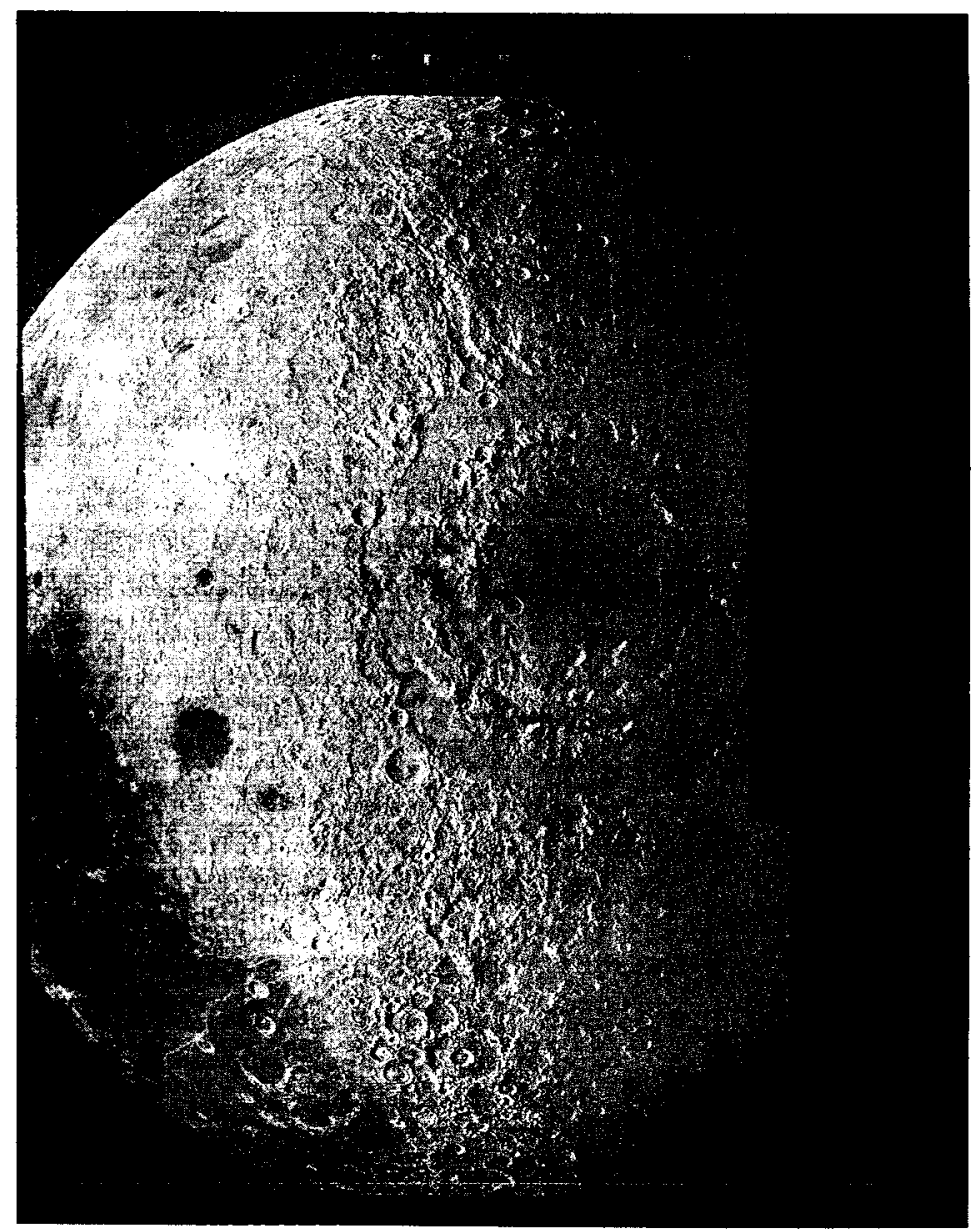

Figure 4: Apollo orbiter view of Mare Orientale, which lies too far around the eastern limb to be seen from earth. This gigantic scar resulted from the collision with the moon of an asteroid perhaps 50 kilometers in diameter. Its vicinity offers some significant advantages for the location of the first lunar outpost. In particular. the very small and dark Mare Grimaldi, in about the 8-o'clock position from Orientale as seen in this picture, is (barely) visible from earth and is one serious candldate for the site. 
aperture in the range of 1-2 meters, to be emplaced below the lunar surface, observing through a well-shielded aperture and using wide-field charge-coupled detectors, like those used in modern TV cameras. Over several years it would yield a unique and invaluable deep study of the ultraviolet and optical sky. The second would be a small, lightweight conventional telescope with high-quality fully automated multichannel photometric recording. It would be necessary only to set this telescope on the lunar surface near the landing site, orient it to the pole, erect a suitable sunshade, then leave it to be operated under command from earth for an indefinite period of time until any maintenance or change in the detectors should be necessary. The system would make long continuous time-series observations of subtle rapid variability of individual stars. Fourier transformation of such data streams yields rich information about stellar seismology, stellar structure, stellar evolution, even evidence bearing on the age of the galaxy.

The entire system should be adapted to the low lunar gravity and total lack of wind, with all its microchip electronics modulized for simple replacement in case of failure. A complete telescope of this type should weigh only a few tens of kilograms, yet can be large enough to do important science for decades. In addition, it would serve as an extremely important test-bed on which to base the design of the next generation of more substantial optical and infrared telescopes to be placed on the moon.

This prototype telescope should be instrumented to measure how well its design copes with the sharp differences between lunar day and night temperatures, the degree to which its nighttime cooling is suitable for infrared work without cryogens, the effectiveness of its shielding against sunlight scattered from the lunar surface during lunar daytime, the rate of accumulation of dust in any critical parts, and the effects of micrometeorites on the exposed optical surfaces. It would not be unreasonable to expect that each supply mission might bring another such low-cost lightweight telescope, leading ultimately to a veritable farm of these simple but valuable systems, with a rich harvest of data for the burgeoning field of stellar seismology and other branches of astronomy.

An early activity of the pioneering lunar base will be exploration of the vicinity with rover vehicles of a more sophisticated class than the little dune buggies that the Apollo mission took to the moon, or the Soviet remote Lunikhod vehicles. As soon as these have demonstrated the ability to make 
long traverses, it might be time to begin installing elements of a very-low-frequency radio astronomy array. At remarkably low cost and with only a small burden on lunar payloads, such a radio telescope could be laid out on the moon. It would open up the essentially unknown spectral region from 1 to 10 megahertz (30-300-meter wavelength band) for relatively high-resolution studies of galactic structure, the low-frequency end of extragalactic phenomena, and allow the search for Jupiter-like radio-emitting objects in other solar systems. The system, proposed by Douglas and Smith in 1987 and studies in some detail at a 1988 Albuquerque workshop on the subject (Burns et al., 1989), will also be a pioneering instrument with which to gain experience in planning large-scale low-frequency radio astronomy developments on the back side of the moon.

Another early instrument should be a meter-class all-reflecting Schmidt camera, designed to produce high-resolution images over wide fields of view at all wavelengths that its mirrors could reflect, but initially concentrating on the ultraviolet sky, which is blocked from terrestrial view by the ozone layer.

A relatively large astronomical construction project, probably to be undertaken in the later phases of the pioneering years, might be to install a telescope concentrating on the thermal infrared, again optimized for light weight but especially for maximum insulation from sources of heat. Such a telescope, of at least the 3-meter class, would require 4-K liquid helium cooling for the detectors, and might experiment with recirculation of helium through critical telescope members or a surrounding jacket. With the low temperature automatically reached by the telescope, the loss of helium would be small and easy to resupply by the adjacent selenauts.

The most important follow-on optical instrument, with designs to be finalized after experience with the initial telescope or telescopes, should be the first lunar optical and infrared interferometer (Burke 1990). This would involve at least a pair, preferably soon an array, of carefully designed telescopes to be spaced out over distances of tens to hundreds of meters, feeding a phase-correcting optical system. While returning invaluable data, this unit would also serve as a prototype for the design of a true optical VLA (very large array)-probably the most important class of scientific instrument ever to be built on the moon. Such arrays will ultimately-perhaps 50 years from now-allow us to study details of structures tens of thousands of times smaller than those accessible to the Hubble Space Telescope. It is probable that 


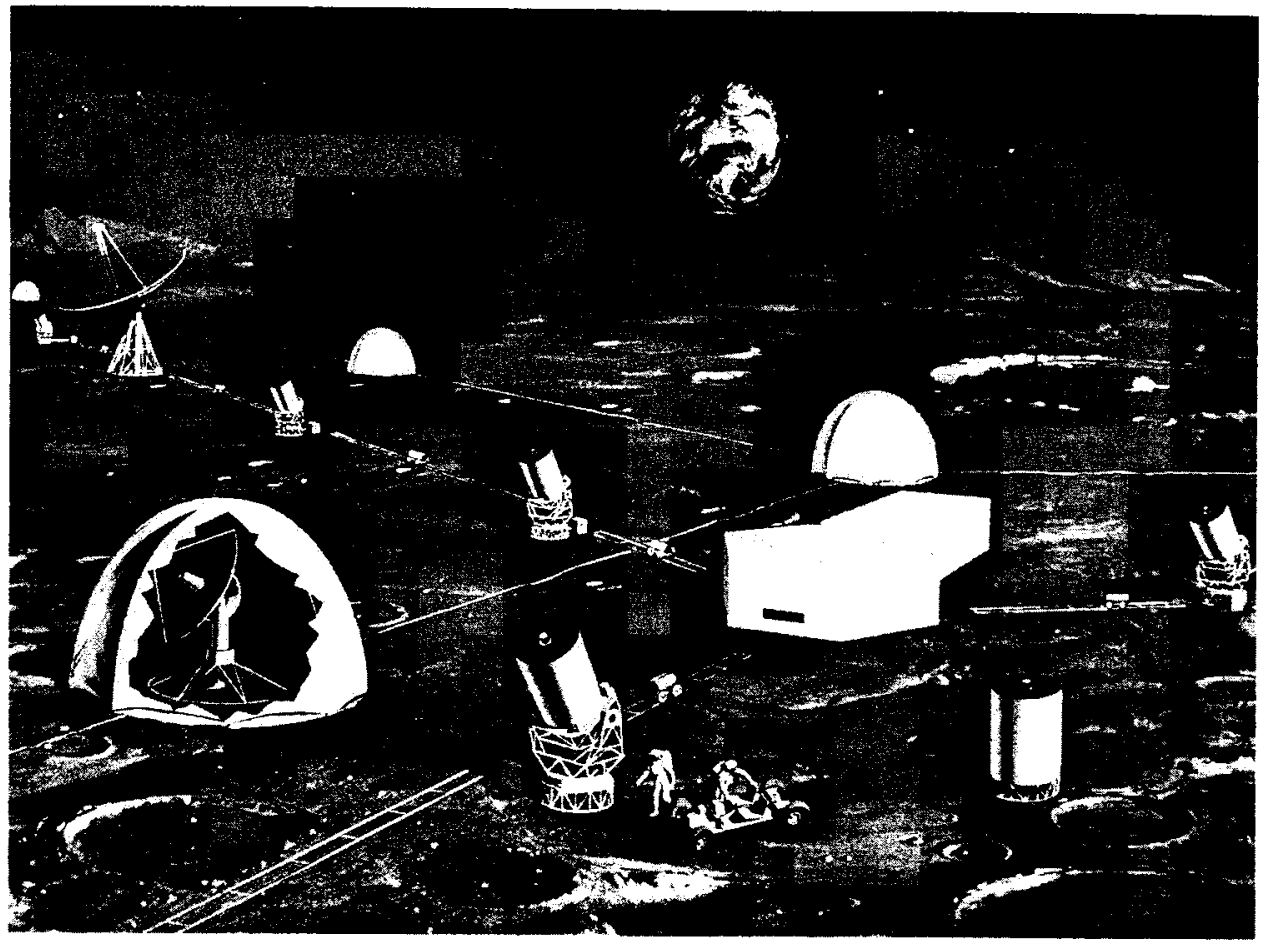

Figure 5: Schematic general appearance of part of a possible lunar observatory perhaps two decades after the permanent return to the moon. A number of relatively small telescopes will serve individual functions, some of them also connected for combined use as an interferometer.

Source NASA

instruments of such prodigious resolving power can in practice be built and operated only on the moon.

Developments such as those so far outlined would lead to the creation of the first true lunar observatory. Major terrestrial observatories need a number of telescopes of various sizes and functions. The heavens are so indescribably rich in objects and phenomena to study that even the smallest telescopes continue to have vital roles to play, for example in following changes of the activity of bright stars. The lunar observatory-with its essentially perfect site conditions for astronomy-can be expected to exemplify this to an extreme degree, continuing to use earlier instruments but constantly accreting new ones initially still of modest size but with increasingly dedicated roles, all under real-time operational control from the earth. 


\section{Consolidation Phase}

The pioneering phase, including establishment of this versatile lunar observatory, should be completed within the first decade of lunar occupation. It should evolve smoothly into a consolidation phase, characterized by a steadily growing population of perhaps dozens rather than a handful of selenautswith reliable life-support and lunar transportation systems, a large amount of power available, no doubt many robots able to work effectively on the lunar surface under teleoperated control of experts in comfortable shirt-sleeve proximity, and the beginning of significant utilization of lunar resources.

During the consolidation phase it may be possible to create a large (kilometer-scale) IR/optical VLA as the keystone of this lunar observatory. Until

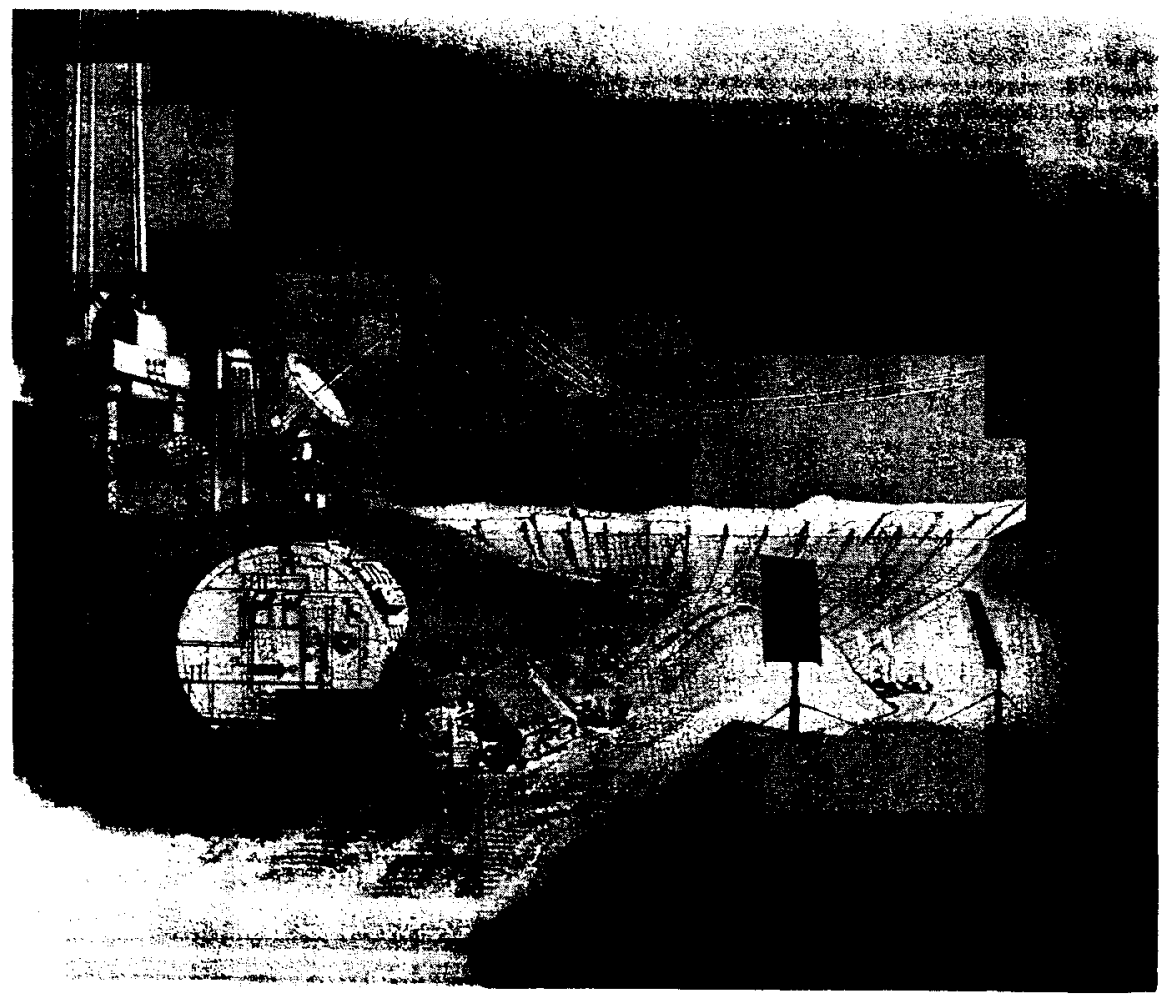

Figure 6: Construction of a very large radio telescope on the lunar back side sometime after the middle of the 21 st century. Most of the materials used will have been produced on the moon. with almost all of the labor performed robotically under close human control. 
this point the lunar telescopes have been complementing other terrestrial and space-based researches, as well as developing the experience needed to design instruments best adapted to lunar conditions. The IR/optical VLA should break truly new ground-being able in principle to look into the hearts of quasars, even able to study details of planetary systems around other stars.

The installation of very large (10-meter class) telescopes should by this point have become feasible. Here again their performance-especially in spatial resolution and wavelength coverage-will be so spectacularly much better than that of their ground-based counterparts as to constitute a genuine scientific breakthrough. Other large instruments for radio and high-energy astronomy as well as other branches of cosmic physics are likely to be proposed for this period. In particular, the back side of the moon by then may have become accessible for installation of the first large radio telescopes in that precious zone of radio quiet.

\section{Settlement Phase}

Several decades further should see the consolidation phase merging into the third stage of lunar development, which might be termed the settlement phase. This will be characterized by easy and low-cost access, relatively substantial populations, almost unlimited power, and major utilization of lunar resources. It will then be possible to undertake truly gigantic astronomical installations, constructed almost entirely by robot labor. Although terrestrial ground-based observatories and specialized space observatories will continue to be operated, it is likely that by the middle of the next century the moon will have become the principal astronomical resource for the human race. Two recent NASA workshops have led to major books detailing these potential developments (Potter and Morgan, 1990; Mumma and Smith, 1990).

\section{INTERNATIONAL SPACE ACTIVITY}

Either the US or the USSR alone could in principle readily accomplish substantial lunar-base development. The required expenditures would be only a few percent of what either country now allots to its military. Nevertheless, it is unlikely that either country will try to do it alone. Such an attempt might even be counterproductive. 
At least five classes of reason support the internationalization of this lunar-base effort:

- Many other countries, including Europe (collectively), China, Japan, India, Israel, and Brazil, already have or soon will have substantial space-based activities.

- Whatever the costs of lunar bases, any nation's budgeters will happily divide the costs with other nations.

- While large-scale international cooperation involves many headaches, when done constructively the results are gratifyingly impressive; for example, CERN and ESO (the European Southern Observatory).

- It is obvious that no single development can redirect the real and imagined mutual suspicions and animosities between the major political blocs in the world today. Nevertheless, the future is likely to be less hazardous if these blocs have been able to cooperate in great and nonthreatening enterprises.

- As has often been noted, the moon is and should remain the heritage of all mankind-developed and used in many different ways by many different groups, but in a spirit of open cooperation.

What would be involved in the proposed cooperation? According to most dictionaries, the verb "to cooperate" in the English language has at least three meanings. Each brings a different nuance to the possible expression of international cooperation as discussed here.

The first meaning, "to operate jointly," is the most literal definition of the word. This idea might apply, for example, to eventual joint piloted missions to Mars where the transportation elements and crew could be totally integrated. The next meaning, "to act together," implies separate elements whose missions complement or supplement each other. An excellent example is the cooperative study of Halley's Comet by the fleet of Soviet, European, and Japanese spacecraft as well as an international array of ground-based observers. Finally, the word can mean "to associate with others for mutual benefit." Mutually accepted treaties or legal regimes fall into this category, which also includes scientific or professional associations where information is freely exchanged. 
The definitions have been ordered in decreasing complexity of the organization required to maintain the cooperation. The order is also one of increasing independence of choice of objectives and modes of operation by the cooperating parties. While pedantic, these distinctions are important to identify because the phrase "international cooperation" may be used in different languages and cultures with connotations that are not readily translated.

Obviously, different forms of cooperation are appropriate in different circumstances. The question here is what forms of international cooperation should be pursued at the highest levels in order to assure the quickest and most complete access to the space frontier for the benefit of all mankind-initially, in particular, to the development of manned lunar bases.

\section{A STRATEGY OF DEVELOPMENT}

Cooperation in the fullest sense would involve the formation of a worldwide international body, analogous to CERN, charged with establishing and operating lunar bases. Such an organization would need a budget, contributed to by the participating countries, and would have the right to negotiate for required services with at least the participating if not indeed all, countries. Although we believe this approach to be desirable in terms of best sharing the costs and most significantly contributing to defuse great-power animosities, it is not likely to be the fastest or least expensive road to lunar development. Nor, given even the present reduced levels of mutual paranoia, is it likely to be achievable on the necessary time scale.

Conversely, the lowest level of cooperation is analogous to that currently experienced in Antarctica, where US, Soviet, and many other nationally supported stations are now more or less permanently manned. The goals of science and exploration are largely held in common. There is much sharing of information and techniques, also occasional visiting to help break the monotony of small group associations and when needed the provision of emergency help from one station to another. This mode is a possible one for the moon as well. In the long run, as space travel becomes less difficult and as lunar development proliferates, it is in fact likely that a number of separate national stations and settlements will develop, but we question whether this is the best approach for now when questions of cost, technology, time, and safety loom so large. 
At present, programs using humans in space are focusing on low-earthorbit (LEO) stations-the Soviet Mir or the proposed international "Freedom." Earth-to-orbit launch systems supporting these stations are based on chemical propulsion technology, with advanced technologies such as electric propulsion probably useful only in interplanetary flights. Therefore, we can assume that the early piloted missions to the moon will utilize chemical propulsion systems evolved from those we see today. Such missions occurring within the next decade will certainly originate in LEO and most likely will be conducted by national space agencies as expeditionary flights. It is within this framework that we most plausibly will need to work.

What specific intermediate level of cooperation, as defined above, will be appropriate for earliest and most likely development of lunar bases? Details must of course be worked out by appropriate authorities, but it is easy to suggest a possible outline:

- The initial effort might involve two roughly equal independent but collaborating consortiums, one led by the US and including major participation by ESA, Japan, and perhaps other countries as well; the other led by the USSR.

- A common target site for the initial lunar base should be selected as soon as possible, based on current information supplemented by data from at least one lunar orbiter to be flown within the next five or six years:

- While increasing commonality should be sought as the program proceeds, the differences in hardware, administration, and operational procedures-also the reluctance of military authorities on either side to allow very full sharing of hard data on details of some of their more sophisticated technologies-are likely to remain too strong over the next one or two decades to permit complete melding. Accordingly, tasks would need to be divided as most appropriate and most strongly desired by the respective parties. For example, while each country with substantial launch capability might contribute something to the task of boosting ultimately thousands of tons to the lunar surface, if the Soviet program remains the one with the largest low-cost launch capability it might undertake the majority of the work of lifting major payloads into low earth orbit. The US might concentrate on orbital transfer vehicles and lunar landers. While 
sharing in all these activities ESA and Japan might also wish to be concerned especially with the design of lunar habitations. A specific task appealing to Japanese participants might be the development of optimal vehicles for travel and work on the lunar surface. Even at the initial lunar base there could and probably should be a number of separate buildings and activities, one or more of which might be operated by any particular cooperating subgroup partly for its own projects and partly for the general operation. A plausible parallel is offered by the European Southern Observatory, where by pooling the resources of many countries they have succeeded in building the world's largest and soon to be best observatory, yet where individual countries or groups of countries own or operate specific telescopes or domes or other facilities, with only certain major "umbrella" functions held in common.

- The expanding lunar operation can be expected to evolve into increasingly specialized units, for example, for observatory construction, selenological prospecting, mining, production of lunar oxygen or metals or other materials, building of mass-driver launch facilities, setting up of remote bases-yes, even the construction and operation of tourist facilities-to be undertaken by whatever international, national, or commercial interest groups chose to make the investment of money and effort.

\section{SUMMARY AND CONCLUDING REMARKS}

Reliable heavy-lift transportation systems-especially Soviet ones-now exist. The next stage in the human expansion should include several kinds of space station. Here again the Soviets are leading the way, although there is reason to hope that before the end of the century there will be one or more additional stations built by the US, ESA, and Japan. The logical targets of further human expansion into space are manned trips to Mars and the establishment of lunar bases.

As the next century begins, the path to the human visitation and eventual settlement of Mars will become clearer. However, we conclude that the establishment of a manned lunar base represents an alternative path superior to the choice of an immediate expedition to Mars.

Exploration, development, and settlement of both the moon and Mars are 
inevitable. The decision as to which comes first will not depend entirely on scientific arguments. Indeed, from the very beginning it may be a largely political choice. However, a moon base is very much the correct choice for the next step, for the principal reasons of proximity, affordability, much less risk for astronauts, the fact that it is the next step on the learning curve as well as springboard for further internationally cooperative development of space, the real possibility of relatively early economic return, the high probability for subsequent substantial human settlement, and the outstanding science to be done from a moon base.

This vision of the future is no doubt blurred from a bit of what astronomers have long called "bad seeing." But is it unreasonable? Is there a truly better alternative? Nor must it be forgotten that one of the most important byproducts of lunar base activity will be the simultaneous evolution of space hardware, fuel supplies, and experience enabling serious Mars expeditions and bases (rather than touchdown stunts) to be developed with practiced cooperation, within a more reasonable budget, and with a high probability of success. The essential infrastructure will finally be in place to allow the human race to begin to develop its extraterrestrial birthright. If these developments are properly handled, national and power-bloc differences can enrich rather than threaten the future of mankind.

\section{NOTES AND REFERENCES}

B.F. Burke, "Astrophysics from the Moon," Science, 250, 7 December 1990, pp.13651370 .

Jack O. Burns, Nebojsa Duric, Stewart W. Johnson, and G. Jeffrey Taylor, eds., “A Lunar Far-side Very Low Frequency Array," NASA conference publication 3039 (1988).

Jack O. Burns, Nebojsa Duric, G. Jeffrey Taylor, and Stewart W. Johnson, "Observations on the Moon," Scientific American, 262, 3 March 1990, pp.42-49.

J.N. Douglas and H.J. Smith, "A Very Low Frequency Radio Astronomy Observatory on the Moon," in W. Mendell ed., Lunar Bases and Space Activities of the 21st Century (Houston, Texas: Lunar and Planetary Institute, 1985), pp. 301-306.

S.W. Johnson and J.P. Wetzel eds., Engineering, Construction and Operation in Space, (New York: American Society of Civil Engineers, 1988).

W.W. Mendell ed., Lunar Bases and Space Activities of the 21st Century (Houston, Texas: Lunar and Planetary Institute, 1985). 
M.J. Mumma and H.J. Smith eds., Astrophysics from the Moon, proceedings of NASA workshop, Annapolis, Maryland, 1990, Conference Proceedings 207 (New York: American Institute of Physics).

A.F. Potter and T.L. Wilson eds., Physics and Astrophysics from a Lunar Base, proceedings of NASA workshop, Stanford, California, 1989, Conference Proceedings 202 (New York: American Institute of Physics).

Ride, S. 1987, "NASA Leadership," A Report to the Administrator, p.54.

G.D. Taylor, "Geological Considerations for Lunar Telescopes," in Future Astronomical Observatories on the Moon, NASA Conference Publication 2489 (1988). 